\title{
Long-term stellar variability
}

\author{
Isabella Pagano ${ }^{1}$ \\ ${ }^{1}$ INAF - Osservatorio Astrofisico di Catania, v. Santa Sofia 78, p.le M. Rodonò, 95123 Catania \\ email: isabella.pagano@oact.inaf.it
}

\begin{abstract}
Stars with significant subsurface convection zones develop magnetic loop structures that, arising from the surface upward to the external atmospheres, cause flux variability detectable throughout the whole electromagnetic spectrum. In fact, diagnostics of magnetic activity are in radio wavelengths, where gyrosincrotron radiation arises from the quiescent and flaring corona; in the optical region, where important signatures are the Balmer lines, the Ca II IRT and H\&K lines; in the UV and X ray domains, the latter mainly due to coronal thermal plasma. The zoo of different magnetic features observed for the Sun - spots, faculae, flares, CMEs - are characterized by different temporal evolution and energetics, both in quantity and quality. As a consequence, the time scale of variability, the amount of involved energy and the quality of the involved photons are used as fingerprints in interpreting the observed stellar variability in the framework of the solar-stellar analogy. Here I review main results from long-term multiwavelength observations of cool star atmospheres, with emphasis to similarities and differences with the solar case.
\end{abstract}

Keywords. stars: activity, stars: spots, stars: chromospheres, stars: coronae, stars: mass loss

\section{Introduction}

Active regions in the atmosphere of late-type stars are the obvious manifestations of surface magnetic activity. Strong magnetic flux ropes are generated by the coupling between differential rotation and turbulent convection in the subphotospheric layers thanks to a "dynamo" mechanisms (see Parker 1955). The flux tubes buoyantly rise through the convection zone and penetrate the stellar surface. By interacting with the surface velocity fields, they become braided, twisted, and ultimately, reconnect releasing magnetic energy that power the atmospheric activity.

The whole phenomenology is higly variable in time. Active regions grow and evolve in days-weeks, but are also site of transient phenomena like flares, microflares, and coronal mass ejections (CMEs). The global level of magnetic activity changes in time following cycles as it happens for the Sun.

The covering factor of active regions, as well as the enery output of stellar flares, can be order of magnitude more intense in stars than on the Sun. This is particularly true for very fast rotators such as young stars and stars locked in close binary systems. The time scale of variability, the amount of involved energy and the quality of the involved photons are used as fingerprints in interpreting the observed stellar variability in the framework of the solar-stellar analogy. Multiwavelength observations are mandatory tools for these investigations. Here, I review some of the results obtained by long-term multiwavelength observations of cool star activity. 


\section{Sources of long-term data on magnetic activity}

Stellar magnetic activity has been detected in all late type stars cooler than $\mathrm{T}_{\text {eff }} \sim$ $8300 \mathrm{~K}$, i.e. later than A4 V (Simon et al. 2002). For typical characteristics these can be grouped as following:

- Sun-like stars: this is a very broad class including from late F to late G type dwarfs and subgiants. Following Cayrel de Strobel \& Bentolila (1989), solar-like stars are usually classified as:

- Solar analogs: these are pop I dwarfs having main properties similar to those of the Sun;

- Solar twins: which have all physical parameters almost identical to those of the Sun.

- M-type stars;

- Rapidly rotating stars: i.e., stars in close-binaries (e.g., BY Dra, RS CVn, FK Com) and young stars.

How magnetic activity changes during the evolution of solar-like stars and K-M dwarfs is of paramount interest to understand properties and evolution of exo-planetary systems. Stellar magnetic activity studies require long term monitoring projects both from the ground and space. Among the observing programs providing bulks of fundamental data for stellar magnetic activity studies I recall the Mount Wilson Observatory Ca II H\&K (e.g., Baliunas et al. 1998), and the Lowell \& Fairborn Observatories (e.g., Radick et al. 1998, Messina \& Guinan 2002 \& 2003, Lockwood et al. 2007) projects, both aimed to the detection of chromospheric variability in solar-like stars, the latter dedicated also to observations of the photospheric signatures of magnetic activity. Photometric and spectroscopic monitoring have been carried out in several European observatories and institutes to get maps of spotted photospheres (e.g., Rodonò 1992, Rodonò \& Cutispoto 1994, Lanza et al. 1998, Rodonò et al. 2000, Messina 2008, Vida et al. 2007; Strassmeier 2009), and of magnetic field patterns (e.g., Donati et al. 2003a, Donati et al. 2003b, Donati et al. 2007). Recently, space observations from MOST, CoRoT, and Kepler are providing very accurate optical variability data with high cadence and months duration (e.g., Walker et al. 2007 \& 2008, Lanza et al. 2009a\&b). Photometric and spectroscopic patrol of stellar flares from the ground have been carried out since the sixties in many places (cf., Gershberg 2005 and references therein).

Finally, the active regions and flares in the upper chromosphere, transition regions and coronae as well as solar-like winds have been studied thanks to the UV, and EUV data from, e.g., IUE, HST, GALEX, EUVE and FUSE (e.g., Jordan et al. 1987; Ayres 1995, Pagano et al. 2004, Ribas et al. 2005, Wood et al. 2005), X-ray data from, e.g., Einstein, Rosat, ASCA, Beppo-SAX, Chandra, and XMM (e.g., Favata \& Micela 2003 and reference therein, Telleschi et al. 2005, Stelzer 2007), and radio observations from VLA, VLBI, VLBA, and single-dishes (e.g., Güdel \& Benz 1993, Benz \& Güdel 1994, Umana et al. 1995, Trigilio et al. 1998).

\section{Results from long-term observations}

\subsection{Photospheres and Chromospheres}

\subsubsection{Solar-like stars}

Ca II H\&K emission data from Mount Wilson Observatory (MWO) have been analyzed together with Strömgren $b, y$ photometry from Lowell and Fairborn Observatories (L\&FO) to investigate the photosphere vs. chromosphere variability of a wide sample of solar-like stars over 1-2 decades time extension (e.g., Lockwood et al. 2007, Hall et al. 
82885

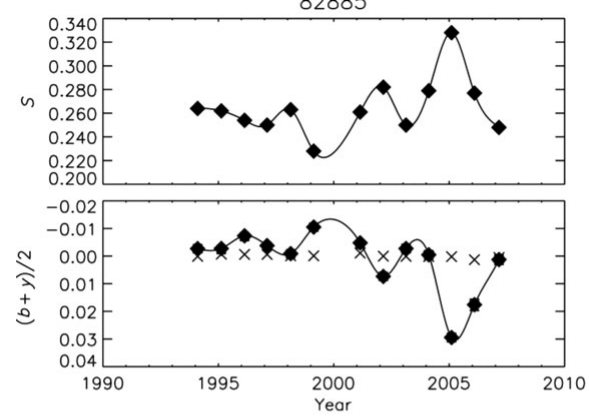

146233

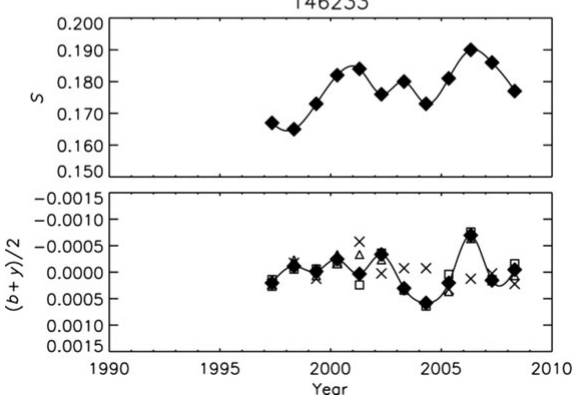

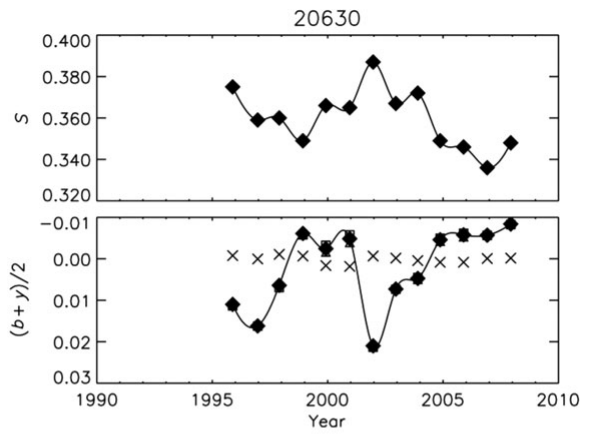

90508

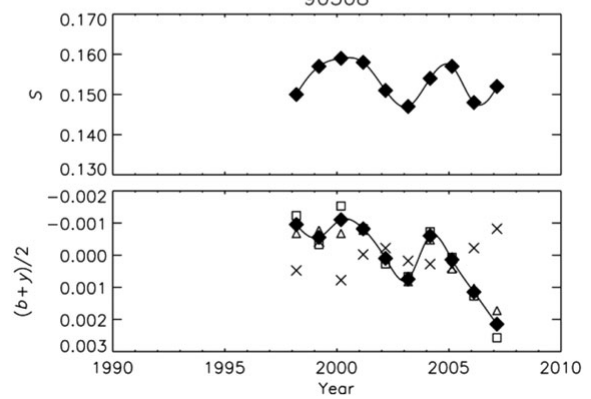

Figure 1. CaII H\&K (top panels) and photometric (bottom panels) series for HD 82885 (G8 IVV), HD 20630 (G5 V), HD 146233 (G2 V), HD 90508 (G0 V) from Hall et al. (2009). Diamonds are the seasonal means. The differential series of the comparison stars are shown by $\times$ symbols.

2009). An important result from these investigations is that, on a year-to-year timescale, young active stars become fainter when their CaII emission increases; i.e., their chromospheric and photospheric light curves are unticorrelated as in the case of HD 82885 and HD 20630 (c.f., Figure 1). Instead, older less active stars, such as the Sun, become brighter when their CaII emission increases, i.e., their chromospheric and photospheric light curves are correlated as in the case of HD 146233, and HD 90508 (cf. Figure 1). In other words, young active solar-like stars are spot-dominated while older less active stars, like the Sun, are faculae dominated. Hall et al. (2009) highlight that the Sun is not peculiar among other solar-like stars; its median photospheric variability is above the one of the solar analogs having chromospheric activity given by $\log R_{H K}<-4.8$. For instance, 18 Sco $(\mathrm{G} 2 \mathrm{~V})$, one of the closest solar twins, with a photometric activity cycle of $7 \mathrm{yr}$, exhibits a chromospheric variability very similar to the solar one, with $\sigma\left(\log R_{H K}\right)=$ 0.043 from 1994 to 2008, that is comparable to 0.040 for the Sun over the same period. Data from MWO and L\&FO have been used to search for stars in Mounder-like magnetic minimum state. Saar (2006) found that the minimum $\mathrm{R}_{H K}$ observed in dwarfs is a decreasing function of stellar metallicity. This effect is well shown in Figure 2-left where the chromospheric activity indicator $\mathrm{R}_{H K}$ vs. $[\mathrm{M} / \mathrm{H}]$ is plotted for the sample of solar like stars analyzed by Hall et al. (2009).

\subsubsection{Rapidly rotating stars}

Rapidly rotating stars show optical light curves changing on time scales of the order of their rotational period due to rotational modulation of starspots and on longer times due to magnetic cycles. From rotational modulated light curves and from the analysis of changing line profiles during the stellar rotation, maps of the spotted photospheres 

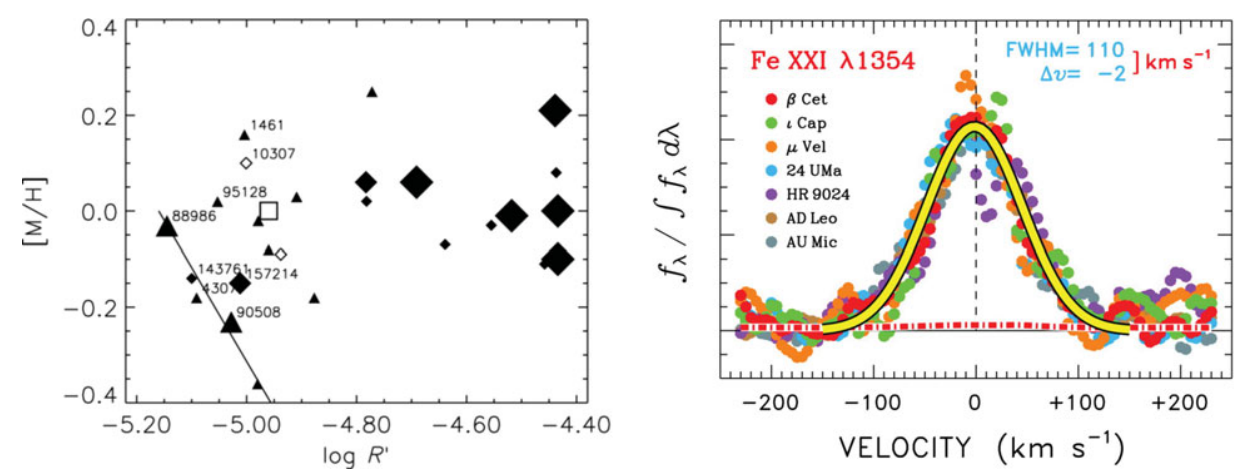

Figure 2. Left: $R_{H K}$ vs. metallicity for a sample of solar-like dwarfs from Hall et al. (2009). The most inactive stars fall on a locus of increasing chromospheric activity with decreasing metallicity. Right: Superposition of scaled Fe XXI $\lambda 1354$ profiles of seven stars, mostly G1-K0 giants but including two M dwarfs. The individual line shapes collectively define a "universal" profile that can be matched by a Gaussian with FWHM $110 \mathrm{~km} \mathrm{~s}^{-1}$ and an essentially negligible $2 \mathrm{~km} \mathrm{~s}^{-1}$ blueshift with respect to the predicted average photospheric velocities of the stars and the adopted wavelength of the Fe XxI $\lambda 1354$ (from Ayres et al. 2003).

and of the magnetic field distribution have been derived by several authors. Among the most noticeable results there are the observations of high-latitude spots (see Güdel 2008, and references therein), and the high-latitude rings of toroidal field detected by Zeeman Doppler imaging (e.g., Donati et al. 2003b,Catala et al. 2007). Also very solar un-like behavior, such as migration of active regions toward the poles, preferred active longitudes, and "flip-flop" effect (e.g., Rodonò et al. 2000, Berdyugina \& Henry 2007) are typically observed.

Messina (2008) has analyzed brightness and color variations in the U, B and V bands of a sample of magnetically active close binary stars on a long time scale, finding that some of them become redder when they are fainter, i.e., as is expected from the presence of active regions mainly consisting of cool spots. Others become bluer when they become fainter, i.e., when spots reach the maximum coverage. In such a case hot photospheric faculae dominate the color variation. Again, this could be interpreted in the framework of the scenario proposed by Radick et al. (1998), Lockwood et al. (2007), and Hall et al. (2009) in which young and rapidly rotating stars arrange their surface magnetic flux predominantly into dark spots, whereas, when stars age and their rotation slows down, bright facula-like structures are favored.

Faculae, plage and supergranulation on the Sun are present even at the minimum of the cycle when few or no spots are on the disk. These features carry a large fraction of the global magnetic flux, but can be elusive at optical wavelength while are best detected in UV where, especially plages, show very large intensity contrasts. Chromospheric and TR maps have been indirectly obtained thanks to time series high-resolution spectroscopy in the UV (e.g. Walter et al. 1987, Neff et al. 1989, Busà et al. 1999, Pagano et al. 2001). One of the main results from these studies is the presence of $\mathrm{T}=10^{4} \mathrm{~K}$ plasma at distances of 1-2 stellar radii from the stellar photospheres. On the other hand, X-ray observations of the same systems reveal that their coronae are filled in with warm $\left(\sim 10^{6} \mathrm{~K}\right)$ and hot $\left(\sim 10^{7} \mathrm{~K}\right)$ plasma components. Hence, the outer atmosphere of rapidly rotating cool stars are site of multi-temperature plasma structures possibly thermally confined by magnetic fields. 


\subsection{Transition Regions and Coronae}

\subsubsection{Plasma dynamics in the transition regions}

Observations of emission lines originating from the TR $\left(10^{5} \mathrm{~K}\right)$ to the corona $\left(10^{7} \mathrm{~K}\right)$ of magnetically active stars have been used to shed light on plasma dynamics, a byproduct of the heating mechanisms at work in the outer atmospheric layers. TR lines observed for the Sun show redshifts increasing with temperature of line formation till about $1-1.2 \times 10^{5} \mathrm{~K}$ (active regions and quiet Sun, respectively). The redshift decreases at higher temperature, crossing over to blueshift at $\mathrm{T} \sim 10^{6} \mathrm{~K}$ (see e.g., Peter \& Judge 1999). A similar effect is observed also for many active stars: $\alpha$ Cen A (G2 V) (Pagano et al. 2004), $\alpha$ Cen B (K1 V) (Wood et al. 1997), and EI Eri (G5 IV) (Jordan et al. 2001) which show the same behavior as the Sun, Procyon (F5 IV-V) that shows a maximum redshift at somewhat higher temperatures (Wood et al. 1997), and $\xi$ Boo A (G8 V) that shows a maximum redshift at temperature lower then typically observed on the Sun (Pagano et al. 2006). The reason of the persistent line shifts has been a challenge for theoreticians; it was generally argued that the dependence of line shifts from temperature of line formation is due to the dynamic response of the atmosphere to the energy deposition after magnetic reconnection events, but several different models have been proposed (e.g., Peter 2006, Spadaro et al. 2006). On the other hand, TR lines of very active dMe stars, like AU Mic (dM1e), certainly show no conspicuous trend of line shift versus formation temperature (Pagano et al. 2000, Redfield et al. 2002). A model capable to represent the case of the Sun and solar analogs, should also explain whether the absence in AU Mic is a consequence of the strong magnetic field, high activity, high gravity, or late spectral type of this target.

Transition region lines are not only redshifted with respect to the photospheric lines, but they also show very broad wings in MS stars, young rapid rotators, and stars in close binaries. For rapidly rotating stars - e.g., AB Dor (Vilhu et al. 1998), or AR Lac (Pagano et al. 2001) - broad wings can arise from rings of hot gas at 2-3 stellar radii, like the "slingshot prominences" seen in H-alpha or the extended loops discussed in Section 3.1.2. For other cases, broad wings can be interpreted as a signature of "microflare" heating (Wood et al. 1997). Redfield et al. (2002) showed that there is a trend of decreasing relative importance of the broad components with increasing line formation temperature, implying that the spectroscopic impact of the mechanism that gives origin to the broad wings might be more important in the lower transition region than at $10^{5} \mathrm{~K}$ temperature. The extension of these investigations to coronal temperatures is challenging, as discussed in section 3.2.2.

\subsubsection{Hi-res spectroscopy of coronal plasma}

Coronal spectroscopy at high resolution has been possible up to date only thanks to the observations of coronal forbidden lines in the UV and FUV spectrum, e.g., Fe XVIII $\lambda$ 974, Fexix $\lambda$ 1118, Fe XII $\lambda$ 1242\&1349, and Fexxi $\lambda 1354$ (c.f., Pagano et al. 2000, Ayres et al. 2003, Redfield et al. 2003). As shown in Figure 2-right for the Fe XXI $\lambda 1354$, centroids of coronal lines of a number of late type giants and $\mathrm{M}$ dwarfs fall at rest with respect to the photosphere, hence the high-temperature plasma is not participating in any large systematic mass motions, but it is likely confined in stable magnetic structures. Moreover, the measured widths of the coronal forbidden lines are generally close to their thermal values (FWHM $40-90 \mathrm{~km} \mathrm{~s}^{-1}$ at $\mathrm{T} \sim 10^{6.2}-10^{7.0} \mathrm{~K}$ ), except for some of the fastest rotating stars in the observed sample (AB Dor, $31 \mathrm{Com}$, and the G1 star of Capella) all showing broadening exceeding the convolution between the thermal and the rotational widths, consistently with excess turbulence or with emitting regions at $\sim 0.4-1.3$ stellar radii from the photosphere. 

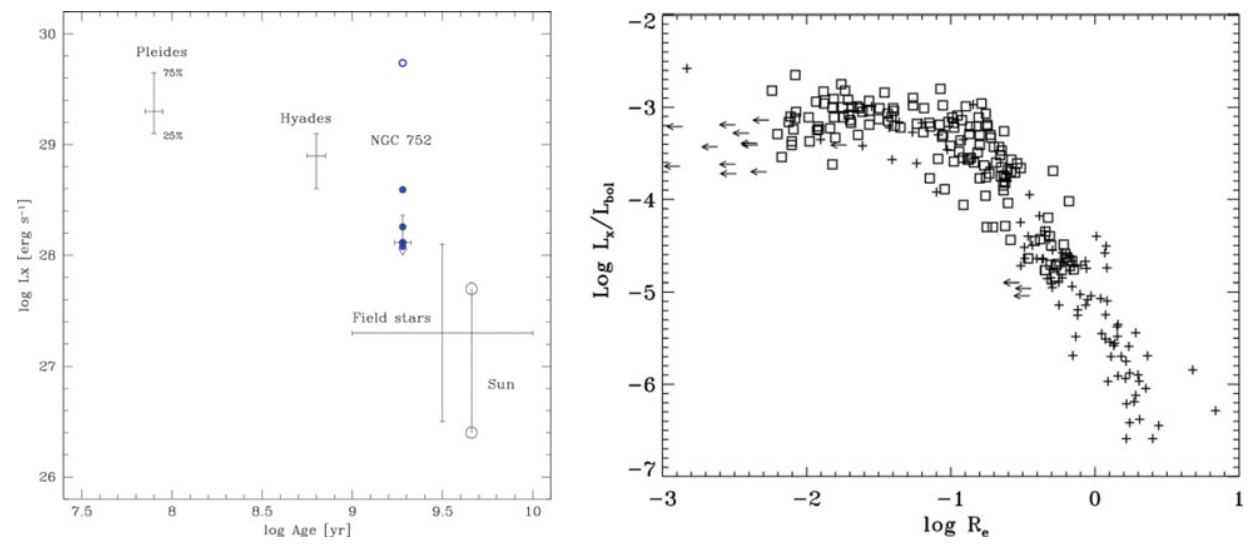

Figure 3. Left: $\mathrm{X}$-ray luminosity of stars with mass in the range $0.8-1.2 \mathrm{M}_{\odot}$ vs. age. The line at age 4.5 Gyr connects the minimum and maximum of the Sun (from Giardino et al. 2008). Right: X-ray to bolometric luminosity ratio vs. empirical Rossby number including field dwarfs (crosses) and cluster stars (squares) (from Pizzolato et al. 2003).

\subsubsection{X-ray emission from coronae}

The soft X-ray flux from stellar coronae is correlated with the Ca II H\&K flux over a factor 100,000 in the X-ray flux (e.g., Schrijver 1987, Ayres 1997), with the mean Sun which obeys the relation and moves along it during the solar cycle. During the stellar life, rotation slows down and consequently the X-ray luminosity decreases by 3-4 orders of magnitude (e.g., Giardino et al. 2008, Güdel 2008). This is not the case of stars in close binaries that, being tidally locked, maintain their fast rotation even at old ages, and therefore have high X-ray luminosity. Rotation period is therefore a good predictor of the total X-ray luminosity, till a critical value below which stars of different masses (or spectral types) enter a saturated regime, corresponding to $\mathrm{L}_{X} / \mathrm{L}_{b o l} \sim 10^{-3}$ at a rotation velocity $\gtrsim 15 \mathrm{~km} \mathrm{~s}^{-1}$ (cf. Figure 3). Pizzolato et al. (2003) have demonstrated that the critical rotation period, below which saturation occours, increases with decreasing stellar mass, suggesting that the X-ray emission in late-type main sequence stars can be considered as an effect depending on the properties of the convective region as well as an effect depending on the total energy budget available in the star.

X-ray luminosity of very fast rotators $\left(\mathrm{v} \sin \mathrm{i}>100 \mathrm{kms}^{-1}\right)$ can be a factor of 3-5 below the saturated level (Randich 1998). The mechanisms responsible for this regime, defined as supersaturation, are not thoroughly understood.

\subsubsection{Rotational modulation of coronal emission}

Stellar rotation periods are generally longer than typical duration of X-ray observations, for this reason rotational modulation of X-ray emission has been detected only sporadically. Unambiguous detections of rotational modulation have been reported by Güdel (1995) for the young solar analogue EK Dra, by Marino et al. (2003) for VXR45, a G9 V star in IC 2391 (cf. Figure 4), by Hussain et al. (2005) for the K0 V AB Dor, and by Stassun et al. (2006) for a number of young stars in the Orion Nebula Cluster. AB Dor as well as many of the stars in ONC showing rotational modulation are fast rotators with an X-ray emission in the saturation regime. VXR45 is even in supersaturation regime. The presence of X-ray rotational modulation in these stars indicates a substantial inhomogeneity in the spatial distribution of active regions. Hence, saturation (and 

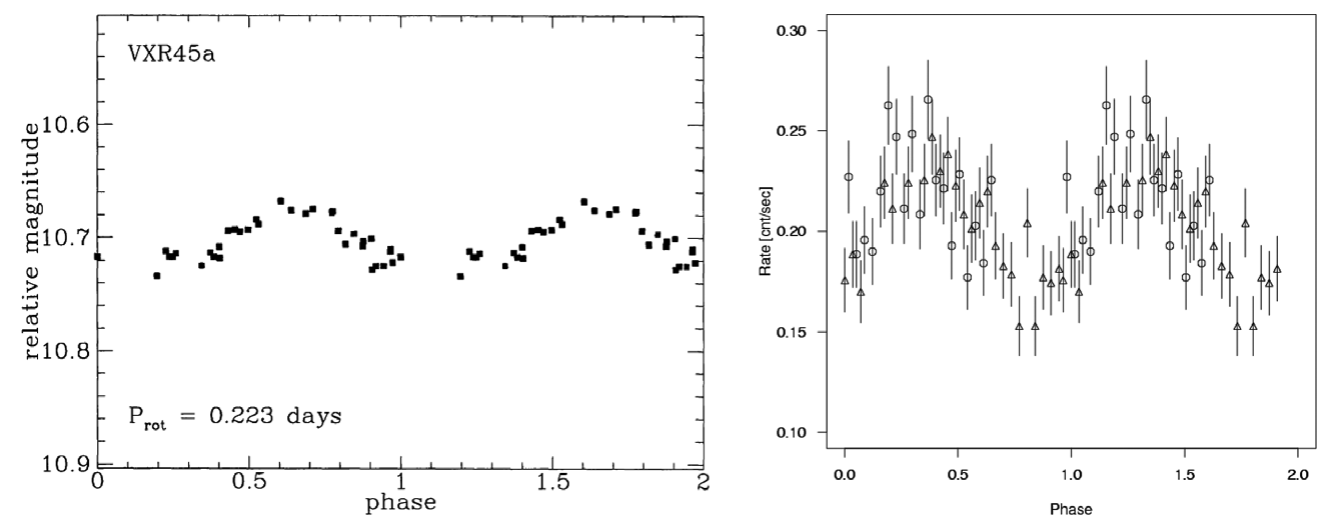

Figure 4. Left: Optical light curve of VXR45a obtained by Patten \& Simon (1996) folded with a period of 0.223 days. Right: X-ray light curve of VXR45a acquired with XMM-Newton in the 0.3-7.8 keV band (see Marino et al. 2003), folded with the same rotation period used for the optical light curve. Circles and triangles have used for data acquired during the first and second covered rotations, respectively.

supersaturation) are not related, at least for these objects, to the filling of the stellar surface with active regions.

\subsubsection{Long-term observations of coronae}

The solar X-ray luminosity follows a cycle with the same periodicity as the chromospheric cycle, but with a much larger amplitude, up to a factor of 100 in the Yohkoh 0.73 - $2.5 \mathrm{keV}$ band (Acton 1996). However, cycles are elusive in other stars. In fact, these have been detected only in $\alpha$ Cen B (Ayres 2009), 61 Cyg (Hempelmann et al. 2006), and HD 81809 (Favata et al. 2008), all solar-like stars with moderate activity. HD 81809 is a close visual binary of slow rotating solar-like stars (G2 V+G9 V) with a well defined chromospheric cycle (Baliunas et al. 1995). XMM-Newton observations have shown a well-defined coronal cycle that has been modeled by Favata et al. (2008) with a simple extension of the solar case in terms of varying coverage of solar-like active regions, but
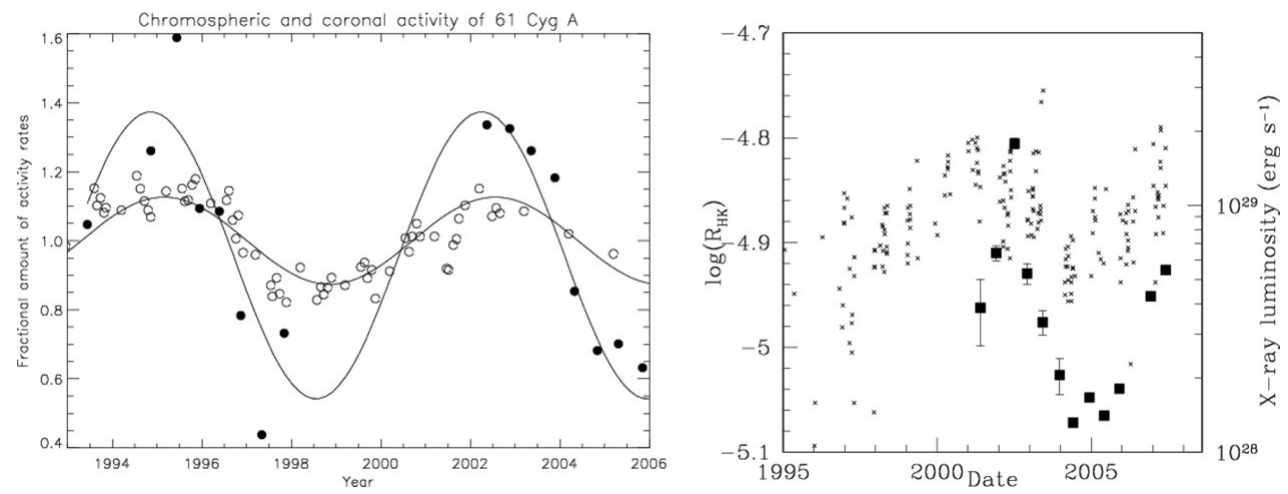

Figure 5. Left: The coronal and chromospheric cycles of 61 Cyg A observed by XMM-Newton (0.2-2.0 keV band) and Mt. Wilson \& Lowell Observatory projects, respectively (see Hempelmann et al. 2006 for details) Right: The coronal and chromospheric cycles of HD 81809 measured thanks to XMM-Newton monitoring in the $0.2-2.5 \mathrm{keV}$ band (squares, right hand scale) and the monitoring of the chromospheric activity index $\mathrm{R}_{H K}$ from Mt. Wilson project (crosses, left hand scale). See Favata et al. (2008) for details. 

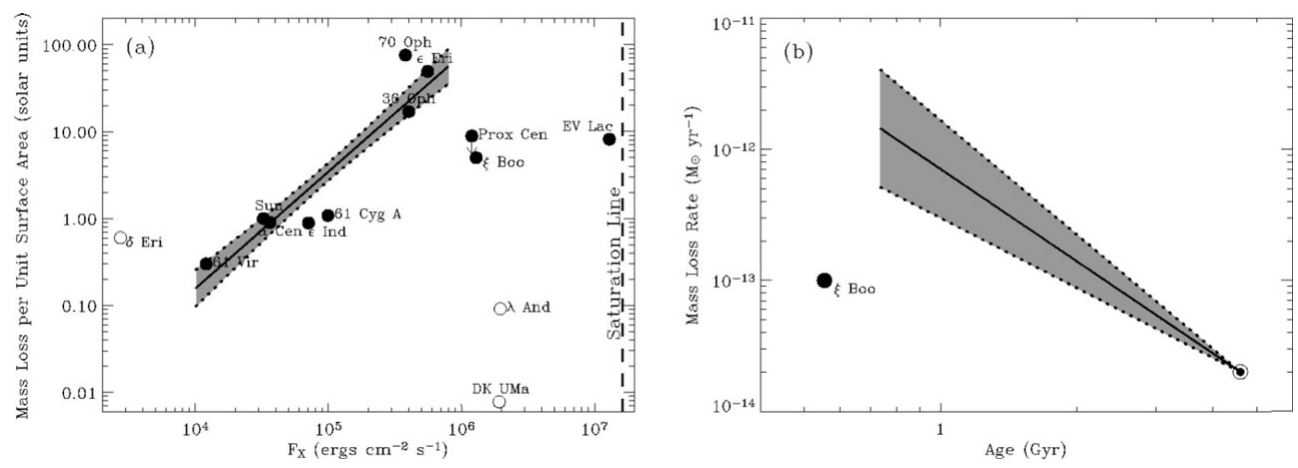

Figure 6. Left: Measured mass loss rates (per unit surface area) plotted versus X-ray surface flux. Right: The mass loss history of the Sun suggested by the power law relation shown in the left panel. Both figures are from (Wood et al. 2005).

with a larger coverage than for the Sun (cf. Figure 6-right). The binary stars in 61 Cyg - K5V (A)+ K7V (B) - observed by XMM-Newton in the X-rays (0.2-2.0 keV) show both short time scales and long-term variations (Hempelmann et al. 2006). In particular, 61 Cyg A has a clear coronal cycle phased with the chromospheric activity cycle (cf. Figure 6-left). The changes of coronal properties during the cycle resemble the solar behaviour. The coronal activity of $61 \mathrm{Cyg} \mathrm{B}$ is more irregular, but also follows the chromospheric activity (Robrade et al. 2007). The close-by $\alpha$ Cen triple system, made by a G2 V (A), a K1 V (B) and the M5V Proxima Centauri, has been monitored by all available X-ray observatories since the '80. Ayres (2009) confirmed the presence for $\alpha$ Cen B of a coronal cycle with period of nine years (as the chromospheric cycle) and a depth of a factor $\sim 6$, comparable to the depth of the solar coronal cycle $(\sim 5)$, while $\alpha$ Cen A did not significantly vary in the $0.2-2.0 \mathrm{keV}$ "ROSAT" band, despite its dramatic fainting in the high energy tail of the coronal spectrum observed by XMM-Newton since 2003 (Robrade et al. 2005 \& 2006). The average coronal luminosity of $\alpha$ Cen B is a factor of 2 higher than that of the Sun, while $\alpha$ Cen A is a similar factor lower, making this star a good candidate as a star in a Mounder-like magnetic minimum. More details on the coronal cycle of $\alpha$ Cen are given by Ayres in this proceedings.

Coronal cycles are not detected in more active stars, possibly because stochastic variability is prevalent in these objects.

\subsection{Winds from late-type dwarfs}

Spectroscopic analyses of stellar H I Ly- $\alpha$ lines have proven to be the best way so far to clearly detect and measure winds of MS stars like the Sun (Wood et al. 2004). In fact, the weak solar-like winds are too hot and thin to provide detectable UV or X-ray absorption (or emission) signatures. Also the wind radio emission is below the sensitivity of radio telescopes in use up to date. In a magnetic active star, Ly- $\alpha$ is in emission and its observed profile is the result of the intrinsic stellar line profile with superimposed the absorptions occurring in the stellar astrosphere (the environment around the star similar to the heliosphere that surrounds the Sun), in the LISM, and finally in the heliosphere. By modeling these absorption features, particularly from the properties of the astrosphere model required to fit the line profile, Wood et al. (2002) performed the first quantitative measurements of mass loss rates for $\mathrm{G}$ and $\mathrm{K}$ dwarf stars, and found that the mass loss rates increase with activity (from X-ray surface flux) and thus with decreasing stellar age. From the observed trend of mass loss rate vs. stellar age it results that the solar wind might have been 1000 times stronger when the Sun was very young, and thereby 
likely played a major role in the evolution of planetary atmospheres, particularly the stripping of volatiles from primitive Mars.

\section{Conclusions}

Stellar variability data tell us that the Sun behaves as a typical solar-likes star in the same phase of evolution. In fact, the Sun activity level and its activity modulation fit very well the behavior of late-type MS stars, in every kind of diagnostic (optical continuum, optical and UV emission lines, X-ray emission). On the other hand, rapidly rotating late-type stars such as young stars and stars in close binary systems show magnetic activity features never observed on the Sun; e.g., polar spots, spot migration towards the poles, and bolometric emission dominated by the cool spots. Preliminary measurements of stellar solar-like winds show that the solar wind could have had a role in the evolution of planetary atmospheres.

New exciting results are expected in the near future from the refurbished HST and EVLA. For instance, the access to UV hi-res (R 20 000) spectra of stars in open clusters will be eventually possible with HST-COS spectrograph, and solar like stellar winds could become directly detectable with the EVLA.

Finally, the understanding of stellar activity phenomena is becoming a must to characterize extrasolar planetary systems, from their discovery to the analysis of their evolution.

\section{References}

Acton, L. 1996, ASP Conf. Ser. 109, p.45

Ayres, T. R., Fleming, T. A., Simon, T., et al. 1995, APJS, 96, 223

Ayres, T. R. 2009, ApJ, 696, 1931

Ayres, T. R. 1997, J. Geophys. Res., 102, 1641

Ayres, T. R., Brown, A., Harper, G.M., et al. 2003, ApJ, 583, 963

Baliunas, S. L., Donahue, R. A., Soon, W. H., et al. 1995, ApJ, 438, 269

Baliunas, S. L., Donahue, R. A., Soon, W., \& Henry, G. W. 1998, ASP Conf. Ser., 154, p. 153

Benz A. \& Güdel, M. 1994, A\& $A, 285,621$

Berdyugina, S. V. \& Henry, G. W. 2007, ApJ, 659, L157

Busà, I., Pagano, I., Rodonò, M., Neff, J. E., \& Lanzafame, A. C. 1999, A\&A, 350, 571

Catala, C., Donati, J.-F., Shkolnik, E., Bohlender, D., \& Alecian, E. 2007, MNRAS, 374, L42

Cayrel de Strobel, G. \& Bentolila, C. 1989, A\& A, 211, 324

Collier Cameron, A., Bedford, D. K., Rucinski, S. M., Vilhu, O., \& White, N. E. 1988, MNRAS, 231,131

Donati, J.-F., Collier Cameron, A., \& Petit, P. 2003a, MNRAS, 345, 1187

Donati, J.-F., Collier Cameron, A., Semel, M. et al. 2003b, MNRAS, 345, 1145

Donati, J.-F., Jardine, M. M., \& Gregory, S. G. 2007, MNRAS, 380, 1297

Favata, F. \& Micela, G. 2003, Space Science Reviews, 108, 577

Favata, F., Micela, G., Orlando, S. et al. 2008, A\&A, 490, 1121

Gershberg, R. E. 2005, Solar-type Activity in Main-Sequence Stars, Springer

Giardino, G., Pillitteri, I., Favata, F., \& Micela, G. 2008, $A \& A$, 490, 113

Güdel, M. \& Benz, A. 1993, ApJ 405, L63

Guedel, M., Schmitt, J. H. M. M., Benz, A. O., \& Elias, N. M. 1995, A\&̋A, 301, 201

Güdel, M. 2008, Living Reviews in Solar Physics, vol. 4, no. 3

Hall, J. C., Henry, G. W., Lockwood, G. W., Skiff, B. A., \& Saar, S. H. 2009, AJ 138, 312

Hempelmann, A., Robrade, J., Schmitt, J. H. M. M., et al. 2006, A\& A, 460, 261

Hussain, G. A. J., Brickhouse, N. S., \& Dupree, A. K. 2005, ApJ, 621, 999

Jordan, C., Ayres, T. R., Brown, A., Linsky, J. L., \& Simon, T. 1987, MNRAS, 225, 903

Jordan, C., Sim, S. A., McMurry, A. D., \& Aruvel, M. 2001, MNRAS, 326, 303 
Kürster, M. \& Dennerl, K. 1993, in Physics of Solar and Stellar Coronae, J. F. Linsky \& S. Serio (eds.), Kluwer Acad. pub., p. 443

Lanza, A. F., Catalano, S., Cutispoto, G., Pagano, I., \& Rodonò, M. 1998, A\&A, 332, 541

Lanza, A. F., Pagano, I., Leto, G., et al. 2009a, A\&A, 493, 193

Lanza, A. F., Aigrain, S., Messina, S., et al. 2009b, A\&GA, in press (arXiv:0901.4618)

Lockwood, G. W., Skiff, B. A., Henry, G. W., et al. 2007, ApJS, 171, 260

Marino, A., Micela, G., Peres, G., \& Sciortino, S. 2003, A\&A, 407, L63

Messina, S. \& Guinan, E. 2002, A\&A, 393, 225

Messina, S. \& Guinan, E. 2003,A\&A, 409, 1017

Messina, S. 2008, A\&\&A, 480, 495

Neff, J. E., Walter, F. M., Rodonò M., \& Linsky, J. L. 1989, A\&A, 215, 79

Pagano, I., Linsky, J. L., \& Carkner, L. 2000, ApJ, 532, 497

Pagano, I., Rodonò, M., Linsky, J. L., et al. 2001, A\&SA, 365, 128

Pagano, I., Linsky, J. L., Valenti, J., \& Duncan, D. K. 2004, A\&A, 415, 331

Pagano, I., De Martino, C., Lanza, A. F., Spadaro, D., \& Linsky, J. L. 2006, ESA-SP 617, 91

Parker, E. N. 1955 ApJ, 122, 293

Patten, B. M. \& Simon, T. 1996, ApJSS, 106, 489

Peter, H. 2006, AdSpR, 39, 1814

Peter, H. \& Judge, P. G. 1999, ApJ, 522, 1148

Pizzolato, N., Maggio, A., Micela, G., Sciortino, S., \& Ventura, P. 2003, A\& A, 397, 147

Radick, R. R., Lockwood, G. W., Skiff, B. A., \& Baliunas, S. L. 1998, APJSS, 118, 239

Randich, S. 1998, ASP Conf. Ser., 154, p. 501

Redfield, S., Linsky, J. L., Ake, T. B., et al. 2002, ApJ, 581, 626

Redfield, S., Ayres, T. R., Linsky, J. L., et al. 2002, ApJ, 585, 993

Ribas, I., Guinan, E. F., Güdel, M., \& Audard, M. 2005, ApJ, 622, 680

Robrade, J., Schmitt, J. H. M. M., \& Favata, F. 2005, A\&A, 442, 315

Robrade, J., Schmitt, J. H. M. M., \& Hempelmann, A. 2007, MSAIt, 78, 311

Rodonò, M. 1992 LNP, 397, 201

Rodonò, M. \& Cutispoto, G. 1994, ASP Conf. Series, 64, 459

Rodonò, M., Messina, S., Lanza, A. F., Cutispoto, G., \& Teriaca L. 2000, A\&A, 358, 624

Saar, S. 2006, BAAS 38, 240

Schrijver, C. J. 1987, ApJ 172, 111

Simon, T., Ayres, T. R., Redfield, S., \& Linsky, J. L. 2002, ApJ, 579, 800

Spadaro, D., Lanza, A. F., Lanzafame, A. C. et al. 2006, ApJ, 582, 486

Stassun, K. G., van den Berg, M., Feigelson, E., \& Flaccomio, E. 2006, ApJ, 649, 914

Stelzer, B., Flaccomio, E., Briggs, K. et al. 2007, A\&\&A, 468, 463

Strassmeier, K. G. 2009, IAU Symp, 259, 363

Telleschi, A., Güdel, M., Briggs, K., et al. 2005, ApJ, 622, 653

Trigilio, C., Leto, P., \& Umana, G. 1998, A\&A, 330, 1060

Umana, G., Trigilio, C.,Tumino, M., Catalano, S., Rodonò, M. 1995, AESA, 298, 143

Vida, K., Kovári, Zs., Ŝvanda, M. et al. 2007, AN, 328, 1078

Vilhu, O., Muhli, P., Huovelin, J., et al. 1998, AJ ,115, 1610

Walker, G. A. H., Croll, B., Kuschnig, R., et al. 2007, ApJ 659, 1611

Walker, G. A. H., Croll, B., Matthews, J. M., et al. 2008, A\&A 482, 691

Walter, F. M., Neff, J. E., Gibson, D. M., et al. 1987, A\&SA, 186, 241

Wood, B. E., Linsky, J. L., \& Ayres, T. R. 1997, ApJ, 478, 745

Wood, B. E., Müller, H.-R., Zank, G. P., \& Linsky, J. L. 2002, ApJ, 574, 412,

Wood, B. E. 2004, Living Reviews in Solar Physics, Vol. 1, no. 2

Wood, B. E., Müller, H.-R., Zank, G. P., Linsky, J. L., \& Redfield, S. 2005, ApJ, 628, L143 